\title{
PAIRS OF QUADRATIC FORMS MODULO ONE
}

\author{
by R. C. BAKER and J. BRÜDERN
}

(Received 23 July, 1991)

1. Introduction. Let $s$ be a natural number, $s \geq 2$. We seek a positive number $\lambda(s)$ with the following property:

Let $\varepsilon>0$. Let $Q_{1}\left(x_{1}, \ldots, x_{s}\right), Q_{2}\left(x_{1}, \ldots, x_{s}\right)$ be real quadratic forms, then for $N>C_{1}(s, \varepsilon)$ we have

$$
\max \left(\left\|Q_{1}(\mathbf{n})\right\|,\left\|Q_{2}(\mathbf{n})\right\|\right)<N^{-\lambda(s)+\varepsilon}
$$

for some integers $n_{1}, \ldots, n_{s}$,

$$
0<\max \left(\left|n_{1}\right|, \ldots,\left|n_{s}\right|\right) \leq N .
$$

Here $\|\theta\|$ denotes the distance from $\theta$ to the nearest integer.

The first result of this kind was obtained by Danicic [6], who showed that one may take

$$
\lambda(s)=\left(3+\frac{4}{s}+\frac{2}{s} \sum_{r=1}^{s} \frac{1}{r}\right)^{-1} .
$$

Thus $\lambda(2)=2 / 13$ and $\lambda(3)=9 / 50$ are admissible. In 1976, however, Schmidt [11] showed that, given real $\alpha, \beta$,

$$
\min _{1 \leq n \leq N} \max \left(\left\|\alpha n^{2}\right\|,\left\|\beta n^{2}\right\|\right)<C_{2}(\varepsilon) N^{-1 / 6+\varepsilon} .
$$

This trivially permits one to take $\lambda(2)=1 / 6$.

Baker and Harman [2] showed that one may take

$$
\lambda(s)=1-\delta(s)
$$

where $\delta(s) \rightarrow 0$ as $s \rightarrow \infty$, although $\delta(s)$ was not calculated explicitly. The method of [2] is weaker than Danicic's for small $s$, but obviously stronger for large $s$.

In the present paper we improve (1.3) for all $s \geq 2$. It is convenient to state our result in terms of the corresponding exponent for a single quadratic form. We write $\alpha(s)$ for a number with the following property: given a real quadratic form $Q\left(x_{1}, \ldots, x_{s}\right)$, then for $\varepsilon>0$ and $N>C_{3}(s, \varepsilon)$ we have

$$
\|Q(\mathbf{n})\|<N^{-\alpha(s)+\varepsilon}
$$

for some integers $n_{1}, \ldots, n_{s}$ satisfying (1.2).

For $s \geq 1$, we may take

$$
\alpha(s)=s /(s+1)
$$

(Danicic [5]). We shall need a generalization of (1.4), which we establish in Section 2. For $s \geq 4$, results stronger than $(1.4)$ have been obtained $[10,3,9]$. In particular, we may take

$$
\begin{gathered}
\alpha(4)=8 / 9[9], \alpha(5)=1[3], \alpha(6)=78 / 71[9], \\
\alpha(s)=2-8 / s[9] .
\end{gathered}
$$

Glasgow Math. J. 35 (1993) 51-61. 
THEOREM 1. We may take

$$
\begin{gathered}
\lambda(s)=\left(2+\frac{6}{s}\right)^{-1} \text { for } s \leq 5, \\
\lambda(s)=\frac{s}{s+11+\alpha(6)^{-1}+\ldots+\alpha(s)^{-1}} \text { for } s \geq 6 .
\end{gathered}
$$

In particular, we may take $\lambda(2)=\frac{1}{5}, \lambda(3)=\frac{1}{4}$.

Clearly the limiting value of $\lambda(s)$ in (1.8) is $2 / 3$; thus [2] is stronger for large $s$. We also observe that stronger results hold for additive quadratic forms [4].

In our proof we use ideas from the lattice method of Schmidt [11], [1]. A key role is also played by estimates for

$$
\sum_{m=1}^{M}|S(m Q)|^{2}
$$

where $Q$ is a real quadratic form and

$$
S(m Q)=\sum_{x_{1}=1}^{N_{1}} \ldots \sum_{x_{s}=1}^{N_{s}} e\left(m Q\left(x_{1}, \ldots, x_{s}\right)\right)
$$

Here $e(\theta)$ denotes $e^{2 \pi i \theta}$. Davenport $[7,8]$ studied the case $M=1, N_{1}=\ldots=N_{s}$ and Danicic [5] treated the case $M>1, N_{1}=\ldots=N_{s}$. We discuss the general case in Section 2.

Constants implied by 《and 》 depend at most on $\varepsilon, s$. We suppose, as we may, that $\varepsilon$ is sufficiently small and write $\delta=\varepsilon^{2}$. We write $|\mathscr{A}|$ for the cardinality of a finite set $\mathscr{A}$. The fractional part of $\theta$ is written $\{\theta\}$.

2. Successive minima. Let

$$
Q\left(x_{1}, \ldots, x_{s}\right)=\sum_{i=1}^{s} \ldots \sum_{j=1}^{s} \lambda_{i j} x_{i} x_{j}
$$

with $\lambda_{i j}=\lambda_{j i}$, and write

$$
L_{i}\left(x_{1}, \ldots, x_{s}\right)=\sum_{j=1}^{s} \lambda_{i j} x_{j} .
$$

Given positive integers $M, N_{1}, \ldots, N_{s}$, we define $S(m Q)$ by $(1.9)(m=1, \ldots, M)$.

Just as on p. 107 of [1], we have

$$
\begin{aligned}
|S(m Q)|^{2} & =\sum_{x_{1}=1}^{N_{1}} \ldots \sum_{x_{s}=1}^{N_{s}} \sum_{1 \leq x_{j}+z_{j} \leq N_{j}} e\left(2 m x_{1} L_{1}(\mathbf{z})+\ldots+2 m x_{s} L_{s}(\mathbf{z})+m Q(\mathbf{z})\right) \\
& \leq \sum_{z_{1}=-\left(N_{1}-1\right)}^{N_{1}-1} \ldots \sum_{z_{s}=-\left(N_{s}-1\right)} \prod_{j=1}^{s} \min \left(\left(N_{j},\left\|2 m L_{j}(\mathbf{z})\right\|^{-1}\right) .\right.
\end{aligned}
$$

In order to estimate the right hand side of (2.1) we define $2 s$ linear forms as follows:

$$
\left.\begin{array}{c}
\zeta_{j}\left(x_{1}, \ldots, x_{2 s}\right)=2 M^{1 / 2} N_{j}\left(L_{j}\left(x_{1}, \ldots, x_{s}\right)-x_{s+j}\right. \\
\zeta_{s+j}\left(x_{1}, \ldots, x_{2 s}\right)=\left(2 M^{1 / 2} N_{j}\right)^{-1} x_{j}
\end{array}\right\} \quad(j=1, \ldots, s) .
$$


Throughout this section, let $\pi_{1}, \ldots, \pi_{2 s}$ denote the successive minima of the lattice

$$
\Gamma=\left\{\left(\zeta_{1}(\mathbf{x}), \ldots, \zeta_{2 s}(\mathbf{x})\right): \mathbf{x} \in \mathbb{Z}^{2 s}\right\}
$$

with respect to the unit ball.

LEMMA 1. We have

$$
1 \ll \pi_{j} \pi_{2 s+1-j} \ll 1 \quad(j=1, \ldots, 2 s) .
$$

Proof. See [8], formula (20).

LemMa 2. Let $B>0$. The number of integer solutions of

$$
\left.\begin{array}{c}
\left\|L_{j}\left(x_{1}, \ldots, x_{s}\right)\right\|<B\left(2 M^{1 / 2} N_{j}\right)^{-1} \\
\left|x_{j}\right|<B\left(2 M^{1 / 2} N_{j}\right)
\end{array}\right\} \quad(j=1, \ldots, s) .
$$

is

$$
\ll 1+\left(\pi_{1} \ldots \pi_{l}\right)^{-1} B^{\prime}
$$

for some $l, 1 \leq l \leq 2 s$.

Proof. The number of solutions of the inequalities (2.3) is at most the number of lattice points $\mathbf{p}$ in $\Gamma$ with $|\mathbf{p}| \leqslant \sqrt{2 s} B$. As in the proof of Lemma 7.1 of [1], the number of such points $\mathbf{p}$ is 1 if $\sqrt{2 s} B<\pi_{1}$ and is

$$
\ll\left(\pi_{1} \ldots \pi_{l}\right)^{-1} B^{\prime}
$$

otherwise, where $l$ is maximal with $\pi_{l} \leq \sqrt{2 s} B$.

Lemma 3. We have

$$
\sum_{m=1}^{M}|S(m Q)|^{2} \ll M^{\delta}\left(N_{1} \ldots N_{s}\right)^{1+2 \delta}\left(1+M^{\prime \prime 2}\left(\pi_{1} \ldots \pi_{l}\right)^{-1}\right)
$$

for some $l, 1 \leq l \leq 2$ s.

Proof. By (2.1), and a standard divisor argument,

$$
\sum_{m=1}^{M}|S(m Q)|^{2} \ll M^{\delta}\left(N_{1} \ldots N_{s}\right)^{\delta} \sum_{x_{1}=1}^{2 M N_{1}} \ldots \sum_{x_{s}=1}^{2 M N_{s}} \prod_{j=1}^{s} \min \left(N_{j},\left\|L_{j}(\mathbf{x})\right\|^{-1}\right) .
$$

Let $k_{1}, \ldots, k_{s}$ be integers satisfying $0 \leq k_{i}<N_{i}$ and let $\mathscr{E}\left(k_{1}, \ldots, k_{s}\right)$ be the set of $\mathbf{x}$ in $\mathbb{Z}^{s}$ with

$$
\frac{k_{j}}{N_{j}} \leq\left\{\begin{array}{l}
\left.L_{j}(\mathbf{x})\right\}<\frac{k_{j}+1}{N_{j}} \\
\left|x_{j}\right|<2 M N_{j}
\end{array}\right\} \quad(j=1, \ldots, s) .
$$

Fix $\mathbf{x}^{\prime}$ in $\mathscr{E}\left(k_{1}, \ldots, k_{s}\right)$. Then for $\mathbf{x}^{\prime \prime}=\mathbf{x}^{\prime}+\mathbf{x}$ in $\mathscr{E}\left(k_{1}, \ldots, k_{s}\right)$,

$$
\left.\begin{array}{c}
\left\|L_{j}(\mathbf{x})\right\|<N_{j}^{-1} \\
\left|x_{j}\right|<4 M N_{j}
\end{array}\right\} \quad(j=1, \ldots, s) .
$$

Applying Lemma 2 with $B=2 M^{1 / 2}$, we obtain 


$$
\max _{k_{1}, \ldots, k_{s}}\left|\mathscr{E}\left(k_{1}, \ldots, k_{s}\right)\right| \ll 1+M^{\prime \prime 2}\left(\pi_{1} \ldots \pi_{l}\right)^{-1}
$$

for some $l, 1 \leq l \leq 2 s$.

Combining (2.5) and (2.6), and writing $k_{j}^{\prime}=\min \left(k_{i}, N-1-k_{j}\right)$,

$$
\begin{aligned}
\sum_{m=1}^{M}|S(m Q)|^{2} \ll M^{\delta}\left(N_{1} \ldots N_{s}\right)^{\delta} \sum_{k_{1}=0}^{N_{1}-1} \ldots \sum_{k_{s}=0}^{N_{s}-1}\left|\mathscr{E}\left(k_{1}, \ldots, k_{s}\right)\right| \prod_{j=1}^{s} \min \left(N_{j}, \frac{N_{j}}{k_{j}^{\prime}}\right) \\
\ll M^{\delta}\left(N_{1} \ldots N_{s}\right)^{\delta}\left(1+M^{\prime}\left(\pi_{1} \ldots \pi_{l}\right)^{-1}\right) \sum_{k_{1}=0}^{N_{1}-1} \ldots \sum_{k_{s}=0}^{N_{s}-1} \prod_{j=1}^{s} \min \left(N_{j}, \frac{N_{j}}{k_{j}^{\prime}}\right) .
\end{aligned}
$$

The lemma follows at once.

We can now prove the generalization of (1.4) mentioned in Section 1.

THEOREM 2. Let $Q\left(x_{1}, \ldots, x_{s}\right)$ be a real quadratic form. Let $N>C_{4}(s, \varepsilon)$ and let $N_{1}, \ldots, N_{s}$ be positive real numbers satisfying

$$
N_{1} \ldots N_{s} \geqslant N^{s} .
$$

Then there are integers $n_{1}, \ldots, n_{s}$, not all zero, satisfying

$$
\begin{gathered}
\| Q\left(n_{1}, \ldots, n_{s} \|<N^{-s /(s+1)+\varepsilon},\right. \\
\left|n_{j}\right| \leq N_{j} \quad(j=1, \ldots, s) .
\end{gathered}
$$

Proof. We consider first the case of positive integral $N_{1}, \ldots, N_{s}$. Suppose if possible that (2.8) has no nonzero solution satisfying (2.9). Let $M=\left[N^{s /(s+1)-\varepsilon}\right]+1$. Applying Theorem 2.2 of [1], together with Cauchy's inequality, we obtain

$$
\sum_{m=1}^{M}|S(m Q)|^{2} \geq M^{-1}\left(\sum_{m=1}^{M}|S(m Q)|\right)^{2} \gg\left(N_{1} \ldots N_{s}\right)^{2} M^{-1}
$$

Combining this with Lemma 3, we have

$$
\left(N_{1} \ldots N_{s}\right)^{2} M^{-1} \ll\left(1+M^{l / 2}\left(\pi_{1} \ldots \pi_{l}\right)^{-1}\right) M^{\delta}\left(N_{1} \ldots N_{s}\right)^{1+2 \delta} .
$$

From (2.2),

$$
\begin{gathered}
\pi_{1} \ldots \pi_{2 s} \ll 1, \\
\left(\pi_{1} \ldots \pi_{l}\right)^{-1} \ll \pi_{l+1} \ldots \pi_{2 s} \ll \pi_{1}^{-(2 s-l)} .
\end{gathered}
$$

Combining (2.10), (2.11), we have

$$
\left(N_{1} \ldots N_{s}\right)^{1-2 \delta} M^{-1-\delta} \ll 1+M^{12} \pi_{1}^{-(2 s-l)} .
$$

From the hypothesis that (2.7) has no solution satisfying (2.8), it follows that

$$
\pi_{1} \geq\left(4 s M^{1 / 2}\right)^{-1} \text {. }
$$

For suppose the contrary; then there is a nonzero integer point $\left(x_{1}, \ldots, x_{2 s}\right)$ with

$$
\begin{aligned}
&\left|L_{j}\left(x_{1}, \ldots, x_{s}\right)-x_{s+j}\right|<\left(2 M^{1 / 2} N_{j}\right)^{-1}\left(4 s M^{1 / 2}\right)^{-1} \\
&<s^{-1} M^{-1} N_{j}^{-1} \\
&\left|x_{j}\right|<2 M^{1 / 2} N_{j}\left(4 s M^{1 / 2}\right)^{-1}<N_{j}
\end{aligned}
$$


for $j=1, \ldots, s$. Now $\left(x_{1}, \ldots, x_{s}\right) \neq \mathbf{0}$, since from $x_{1}=\ldots=x_{s}=0$ one obtains $x_{s+1}=$ $\ldots=x_{2 s}=0$ via (2.14). Thus there is a nonzero integer point $\left(x_{1}, \ldots, x_{s}\right)$ satisfying (2.15) and

$$
\begin{aligned}
\left\|Q\left(x_{1}, \ldots, x_{s}\right)\right\| & =\left\|\sum_{j=1}^{s} x_{j} L_{j}(\mathbf{x})\right\| \\
& \leq \sum_{j=1}^{s}\left|x_{j}\right|\left\|L_{j}(\mathbf{x})\right\|<\sum_{j=1}^{s} N_{j} s^{-1} M^{-1} N_{j}^{-1}=M^{-1},
\end{aligned}
$$

which is a contradiction. This establishes (2.13).

Combining (2.12) and (2.13) yields

$$
\begin{gathered}
\left(N_{1} \ldots N_{s}\right)^{1-2 \delta} M^{-1-\delta} \ll M^{s}, \\
M^{s+1+\delta} \gg\left(N_{1} \ldots N_{s}\right)^{1-2 \delta} \gg N^{s-2 \delta s}
\end{gathered}
$$

from (2.7). This contradicts the definition of $M$, and Theorem 2 is proved in the case of positive integral $N_{j}$.

The case $N_{1} \geq 1, \ldots, N_{s} \geq 1$ follows at once. For (2.7) implies

$$
\left[N_{1}\right] \ldots\left[N_{s}\right] \geq(N / 2)^{s} \text {. }
$$

Since

$$
(N / 2)^{-s /(s+1)+\varepsilon / 2}<N^{-s /(s+1)+\varepsilon}
$$

for large $N$, this permits us to solve (2.8) subject to (2.9) on enlarging $C_{4}$.

We may now prove the general case by induction on $s$. The case $s=1$ has been done. Now let $s \geqslant 2$ and suppose the theorem known for forms in $s-1$ variables. Let $N_{1}>0, \ldots, N_{s}>0$ satisfy (2.7) with $N \geq C_{4}(s, \varepsilon)$. We may suppose some $N_{j}$ is less than 1 ; say

$$
N_{s}<1 .
$$

But then

$$
N_{1} \ldots N_{s-1} \geq H^{s-1}
$$

where $H=N^{s /(s-1)}$. Since $H$ is large, there is a nonzero solution of

$$
\left\|Q\left(n_{1}, \ldots, n_{s-1}, 0\right)\right\|<H^{-(s-1) / s+\varepsilon}
$$

in integers $n_{1}, \ldots, n_{s-1}$ with $\left|n_{j}\right| \leq N_{j}$. Since

$$
H^{-(s-1) s+\varepsilon}=N^{-1} H^{\varepsilon} \leqslant N^{-1+2 \varepsilon},
$$

this completes the induction step, and Theorem 2 is proved.

We may describe the substitution of (2.9) for (1.2) as "replacing a cube by a box". It seems to be difficult to replace a cube by a box in the work of Baker and Harman [3], and more difficult still in the work of Heath-Brown [9]. This is a pity, since such results would lead to an improvement of Theorem 1 for $s \geqslant 4$. 
3. Proof of Theorem 1. Define $\lambda(s)$ by (1.7), (1.8). Let $N>C_{1}(s, \varepsilon)$ and let

$$
\Delta=N^{2(\lambda-\varepsilon)}, \quad \Lambda=\Delta^{1 / 2} \mathbb{Z}^{2}, \quad \Pi=\Delta^{-1 / 2} \mathbb{Z}^{2} .
$$

Let $\Pi^{*}$ denote the set of primitive points of $\Pi$. Let $K_{0}$ denote the unit ball in $\mathbb{R}^{2}$. Let

$$
\mathbf{Q}(\mathbf{x})=\Delta^{1 / 2}\left(Q_{1}(\mathbf{x}), Q_{2}(\mathbf{x})\right)=\sum_{i=1}^{s} \lambda_{i j} x_{i} x_{i}
$$

where $\lambda_{i j}=\lambda_{j i}$. Let

$$
\mathbf{L}_{i}(\mathbf{x})=\sum_{j=1}^{s} \lambda_{i j} x_{j} \quad(i=1, \ldots, s) .
$$

To prove the theorem, it suffices to find $\mathbf{n}$ satisfying (1.2) for which

$$
\mathbf{Q}(\mathbf{n}) \in \Lambda+K_{0} \text {. }
$$

Suppose that there is no such n. Applying [1], Lemma 7.4, we find that

Here

$$
\sum_{\substack{\mathbf{p} \in \mathbf{\Pi} \\ \mathbf{0}<|\mathbf{p}|<N^{s}}}|S(\mathbf{p} \mathbf{Q})| \gg N^{s} \text {. }
$$

$$
S(\mathbf{p Q})=\sum_{n_{1}=1}^{N} \ldots \sum_{n_{s}=1}^{N} e\left(\mathbf{p Q}\left(n_{1}, \ldots, n_{s}\right)\right),
$$

ab denotes dot product, and $|\mathbf{a}|=(\mathbf{a a})^{1 / 2}$.

We may rewrite (3.2) in the form

$$
\sum_{\substack{\mathbf{p} \in \Pi^{*} \\ 0<|\mathbf{p}|<N^{\infty}}} \sum_{m=1}^{[N \delta / \mathbf{p} \mid]} \mid S\left(m \mathbf{p Q} \mid \gg N^{s}\right.
$$

The outer summation is taken over $\ll \Delta N^{2 \delta}$ points $\mathbf{p}$. We select p for which

$$
\sum_{m=1}^{[N \delta /|\mathbf{p}|]}|S(m \mathbf{p Q})| \gg N^{s-2 \delta} \Delta^{-1}
$$

It is helpful to note that

$$
\Delta^{-1 / 2} \leq|\mathbf{p}|<N^{\delta}
$$

We now apply Lemma 3 , taking $Q(\mathbf{x})=\sum_{i=1}^{s} \sum_{j=1}^{s} \mathbf{p} \lambda_{i j} x_{i} x_{j}$ and $L_{i}(\mathbf{x})=\mathbf{p} \mathbf{L}_{i}(x)$; also $N_{1}=\ldots=N_{s}=N$. With successive minima $\pi_{1}, \ldots, \pi_{2 s}$ defined as in Section 2, and with

$$
M=\left[N^{\delta}|\mathbf{p}|^{-1}\right]
$$

we have

$$
\sum_{m=1}^{M}|S(m \mathbf{p Q})|^{2} \ll N^{s+3 \delta s}\left(1+M^{I / 2}\left(\pi_{1} \ldots \pi_{l}\right)^{-1}\right)
$$

for some $l, 1 \leq l \leq 2 s$. In conjunction with (3.3) and Cauchy's inequality, this yields

$$
N^{2 s-4 \delta} \Delta^{-2} M^{-1} \ll N^{s+3 \delta s}+N^{s+3 \delta s} M^{l / 2}\left(\pi_{1} \ldots \pi_{l}\right)^{-1}
$$


In view of (3.4), (3.5), it is easily verified that

so that

$$
N^{s+3 \delta s} \ll N^{2 s-5 \delta} \Delta^{-2} M^{-1},
$$

$$
\pi_{1} \ldots \pi_{l} \ll N^{-s+5 \delta s} M^{1+l / 2} \Delta^{2} .
$$

Suppose for a moment that $l>s$. We apply (2.2). Cancelling $\pi_{2 s+1-l} \pi_{l}, \ldots, \pi_{s} \pi_{s+1}$ from (3.6),

$$
\pi_{1} \ldots \pi_{2 s-l} \ll N^{-s+5 \delta_{s}} M^{1+l / 2} \Delta^{2} .
$$

We deduce that in all cases there is a $k, 1 \leq k \leq s$, such that

$$
\pi_{1} \ldots \pi_{k} \ll N^{-s+5 \delta s} M^{1+s-k / 2} \Delta^{2} .
$$

We now find a lower bound for $\pi_{r}(1 \leq r \leq s)$. By definition of successive minima, there are points $\mathbf{z}_{1}, \mathbf{y}_{1}, \ldots, \mathbf{z}_{s}, \mathbf{y}_{s}$ in $\mathbb{Z}^{s}$ such that $\left(\mathbf{z}_{1}, \mathbf{y}_{1}\right), \ldots,\left(\mathbf{z}_{s}, \mathbf{y}_{s}\right)$ are linearly independent in $\mathbb{Z}^{2 s}$ and, for $j=1, \ldots, s$,

$$
\begin{gathered}
2 M^{1 / 2} N\left|\mathbf{p} \mathbf{L}_{j}\left(\mathbf{z}_{r}\right)-y_{r j}\right| \leq \pi_{r}, \\
\left(2 M^{1 / 2} N\right)^{-1}\left|z_{r j}\right| \leq \pi_{r} .
\end{gathered}
$$

Here $\mathbf{z}_{r}=\left(z_{r 1}, \ldots, z_{r s}\right), \mathbf{y}_{r}=\left(y_{r 1}, \ldots, y_{r s}\right)$.

In particular,

$$
\left\|\mathbf{p} \mathbf{L}_{j}\left(\mathbf{z}_{r}\right)\right\| \ll M^{-1 / 2} N^{-1} \pi_{r} .
$$

According to $[\mathbf{1}]$, Lemma 7.9 , this implies

$$
\mathbf{L}_{j}\left(\mathbf{z}_{r}\right)=\boldsymbol{l}_{j r}+\mathbf{s}_{j r}+\mathbf{b}_{j r}
$$

with $l_{j r} \in \Lambda$ and

$$
\left|\mathbf{b}_{j r}\right| \ll|\mathbf{p}|^{-1} M^{-1 / 2} N^{-1} \pi_{r}
$$

where $\mathbf{s}_{j r}$ lies in the 1-dimensional space $\mathbf{p}^{\perp}$ orthogonal to $\mathbf{p}$.

Consider the points $\boldsymbol{\theta}_{u v}$ defined by

$$
\mathbf{Q}\left(x_{1} \mathbf{z}_{1}+\ldots+x_{r} \mathbf{z}_{r}\right)=\sum_{u=1}^{r} \sum_{v=1}^{r} \boldsymbol{\theta}_{u v} x_{u} x_{v}
$$

By an easy computation,

$$
\begin{aligned}
\boldsymbol{\theta}_{u v} & =\sum_{j=1}^{s} \mathbf{z}_{u j} \mathbf{L}_{j}\left(\mathbf{z}_{v}\right) \\
& =\sum_{j=1}^{s} \mathbf{z}_{u j}\left(\boldsymbol{l}_{j v}+\mathbf{s}_{j v}+\mathbf{b}_{j v}\right) .
\end{aligned}
$$

Consequently, there is a form $\mathbf{Q}_{0}\left(x_{1}, \ldots, x_{r}\right)$ with coefficients in $\mathbf{p}^{\perp}$ such that

$$
\mathbf{Q}\left(x_{1} \mathbf{z}_{1}+\ldots+x_{r} \mathbf{z}_{r}\right) \equiv \mathbf{Q}_{0}\left(x_{1}, \ldots, x_{r}\right)+\sum_{u=1}^{r} \sum_{v=1}^{r} x_{u} x_{v} \sum_{j=1}^{s} z_{u j} \mathbf{b}_{j v}(\bmod \Lambda)
$$

whenever $\mathbf{x} \in \mathbb{Z}^{r}$. 
The one-dimensional lattice $\Lambda^{\prime}=2 \Lambda \cap \mathbf{p}^{\perp}$ has determinant $2|\mathbf{p}| \Delta$, as one easily verifies. By the definition of $\alpha(r)$ we may choose integers $x_{1}, \ldots, x_{r}$ with

$$
\begin{aligned}
0<\max _{u}\left|x_{u}\right| & \ll d\left(\Lambda^{\prime}\right)^{\delta+1 / \alpha(r)} \\
& \ll(|\mathbf{p}| \Delta)^{\delta+1 / \alpha(r)}
\end{aligned}
$$

such that

$$
2 \mathbf{Q}_{0}\left(x_{1}, \ldots, x_{r}\right) \in \Lambda^{\prime}+K_{0} .
$$

In particular, we have

$$
\mathbf{Q}_{0}\left(x_{1}, \ldots, x_{r}\right) \in \Lambda^{\prime}+\frac{1}{2} K_{0} .
$$

Suppose for a moment that

$$
\sum_{u=1}^{r} \boldsymbol{x}_{u} \mathbf{z}_{u}=\mathbf{0}
$$

Then, recalling (3.12), (3.8), we have

$$
\begin{aligned}
\left|\sum_{u=1}^{r} x_{u} y_{u j}\right| & =\left|\sum_{u=1}^{r} x_{u}\left\{\mathbf{p} \mathbf{L}_{j}\left(\mathbf{z}_{u}\right)-y_{u j}\right\}\right| \\
& \ll(|\mathbf{p}| \Delta)^{\delta+1 / \alpha(r)} M^{-1 / 2} N^{-1} \pi_{r}
\end{aligned}
$$

for $j=1, \ldots, s$. Since $\left(\mathbf{z}_{1}, \mathbf{y}_{1}\right), \ldots,\left(\mathbf{z}_{s}, \mathbf{y}_{s}\right)$ are linearly independent, not all the integers

$$
\sum_{u=1}^{r} x_{u} y_{u j} \quad(j=1, \ldots, s)
$$

are zero. It follows that

$$
\pi_{r} \gg M^{1 / 2} N(|\mathbf{p}| \Delta)^{-\delta-1 / \alpha(r)}
$$

Now suppose that

Then either

$$
\sum_{u=1}^{r} x_{u} \mathbf{z}_{u} \neq \mathbf{0}
$$

$$
\left|\sum_{u=1}^{r} x_{u} \mathbf{z}_{u}\right|>N
$$

or

$$
\left|\sum_{u=1}^{r} \sum_{v=1}^{r} x_{u} x_{v} \sum_{j=1}^{s} \mathbf{z}_{u j} \mathbf{b}_{j v}\right|>1 / 2 .
$$

For, if both these inequalities fail, we can combine (3.11), (3.14) to obtain a non-zero integer vector

$$
\mathbf{n}=\sum_{u=1}^{r} x_{u} \mathbf{z}_{u}
$$

satisfying (1.2) and (3.1), which is a contradiction. 
If (3.18) holds then, recalling (3.9), (3.12), we have

$$
\begin{aligned}
& (|\mathbf{p}| \Delta)^{\delta+1 / \alpha(r)} \pi_{r} M^{1 / 2} N \gg N, \\
& \pi_{r} \gg M^{-1 / 2}(|\mathbf{p}| \Delta)^{-\delta-1 / \alpha(r)} .
\end{aligned}
$$

If (3.19) holds, then recalling (3.9), (3.12), (3.10), we have

$$
(|\mathbf{p}| \Delta)^{2 \delta+2 / \alpha(r)}|\mathbf{p}|^{-1} \pi_{r}^{2} \gg 1,
$$

that is

$$
\pi_{r} \gg|\mathbf{p}|^{1 / 2}(|\mathbf{p}| \Delta)^{-\delta-1 / \alpha(r)} .
$$

Taking into account (3.5), we conclude that, in all cases

$$
\pi_{r} \gg|\mathbf{p}|^{1 / 2} N^{-\delta}(|\mathbf{p}| \Delta)^{-\delta-1 / \alpha(r)} .
$$

We can refine the above method to obtain a lower bound for $\pi_{1} \ldots \pi_{r}$ which is useful for small $r$. By Theorem 1, we may choose integers $x_{1}, \ldots, x_{r}$, not all zero, such that

$$
\left|x_{u}\right|<H \pi_{u}^{-1} \quad(u=1, \ldots, r)
$$

and (3.13) holds, provided that $H$ satisfies

$$
H \pi_{1}^{-1} \ldots H \pi_{r}^{-1} \geqslant(|\mathbf{p}| \Delta)^{\delta+r+1} .
$$

We take

$$
H=\left(\pi_{1} \ldots \pi_{r}\right)^{1 / r}(|\mathbf{p}| \Delta)^{\delta+(r+1) / r} .
$$

Suppose now that (3.15) holds. Then from (3.8), (3.21),

$$
\begin{aligned}
\sum_{u=1}^{r} x_{u} y_{u j} & =\sum_{u=1}^{r} x_{u}\left\{\mathbf{p} \mathbf{L}_{j}\left(\mathbf{z}_{u}\right)-y_{u j}\right\} \\
& \ll \sum_{u=1}^{r} H \pi_{u}^{-1} M^{-1 / 2} N^{-1} \pi_{u} \ll H M^{-1 / 2} N^{-1}
\end{aligned}
$$

As in the proof of (3.16) we find that

$$
H \gg M^{1 / 2} N
$$

Now suppose that (3.17) holds. As before, either (3.18) or (3.19) holds. If (3.18) holds, then

$$
\sum_{u=1}^{r} H \pi_{u}^{-1} M^{1 / 2} N \pi_{u} \gg N
$$

from $(3.21),(3.9)$, that is,

$$
H \gg M^{-1 / 2} \text {. }
$$


If (3.19) holds, then

$$
\sum_{u=1}^{r} \sum_{v=1}^{r} H \pi_{u}^{-1} H \pi_{v}^{-1} M^{1 / 2} N \pi_{u}|\mathbf{p}|^{-1} M^{-1 / 2} N^{-1} \pi_{v} \gg 1
$$

from (3.21), (3.9), (3.10). That is,

$$
H \gg|\mathbf{p}|^{1 / 2} .
$$

We deduce that

$$
\pi_{1} \ldots \pi_{r} \gg(|\mathbf{p}| \Delta)^{-r \delta-(r+1)}|\mathbf{p}|^{r / 2} N^{-r \delta}
$$

in all cases.

For $r \geqslant 6$, we can combine (3.24) (with $r$ replaced by 5) and (3.20) (with $6, \ldots, r$ in place of $r$ ). The outcome can be written in a way that incorporates (3.24), namely

$$
\pi_{1} \ldots \pi_{r} \gg(|\mathbf{p}| \Delta)^{-r \delta-\mu(r)}|\mathbf{p}|^{r 2} N^{-r \delta}
$$

for $r \geq 1$. Here $\mu(r)=r+1$ for $r \leq 5$, while

$$
\mu(r)=6+\frac{1}{\alpha(6)}+\ldots+\frac{1}{\alpha(r)}<r+1(r \geq 6) .
$$

We now combine (3.7) with the case $r=k$ of (3.25), obtaining

$$
(|\mathbf{p}| \Delta)^{-k \delta-\mu(k)}|\mathbf{p}|^{k / 2} N^{-k \delta} \ll N^{-s+5 \delta s} M^{1+s-k / 2} \Delta^{2} .
$$

Taking into account (3.4) and (3.5), we deduce that

$$
\begin{aligned}
\Delta^{(s+5+\mu(s)) / 2} & =\Delta^{2+\mu(s)} \Delta^{(s+1-\mu(s)) / 2} \\
& \gg \Delta^{2+\mu(s)}|\mathbf{p}|^{\mu(s)-1-s} \\
& \geq \Delta^{2+\mu(k)}|\mathbf{p}|^{\mu(k)-1-s} \gg N^{s-\varepsilon} .
\end{aligned}
$$

This contradicts the definition of $\Delta$, and Theorem 1 is proved.

\section{REFERENCES}

1. R. C. Baker, Diophantine inequalities, (Oxford University Press, 1986).

2. R. C. Baker and G. Harman, Small fractional parts of quadratic and additive forms, Math. Proc. Camb. Phil Soc. 90 (1981), 5-12.

3. R. C. Baker and G. Harman, Small fractional parts of quadratic forms, Proc. Edinburgh Math. Soc., 25 (1982), 269-277.

4. R. C. Baker and S. Schäffer. Pairs of additive quadratic forms modulo one. Submitted to Acta Arith.

5. I. Danicic, An extension of a theorem of Heilbronn. Mathematika, 5 (1958), 30-37.

6. I. Danicic, The distribution $(\bmod 1)$ of pairs of quadratic forms with integer variables, $J$. London Math. Soc. 42 (1967), 618-623. 81-101.

7. H. Davenport, Indefinite quadratic forms in many variables, Mathematika 3 (1956),

8. H. Davenport, Indefinite quadratic forms in many variables II, Proc. London Math. Soc. (3), 8 (1958), 109-126. 
9. D. R. Heath-Brown. Small solutions of quadratic congruences, II. Mathematika, to appear.

10. A. Schinzel, H. P. Schlickewei and W. M. Schmidt, Small solutions of quadratic congruences and small fractional parts of quadratic forms, Acta Arith. 37 (1980), 241-248.

11. W. M. Schmidt, Small fractional parts of polynomials, Regional Conference Series No. 32, American Math. Soc., Providence 1977.

Department of Mathematics

Royal Holloway and Bedford New College

EGHAM

SURREY TW20 0EX

UK

Mathematisches Institut

GEORG-AUguST-UNIVERSITÄT

BUNSENSTRASSE 3-5

D-3400 GÖTTINGEN

Germany

Present address:

Department of Mathematics

Brigham Young UnIVERSITY

Provo

UTAH 84602

U.S.A. 